\title{
Asylunwürdigkeit wegen «Gefährdung der inneren oder äusseren Sicherheit»: von abstrakter Gefährdung und Beweislastumkehr
}

Kommentierung ausgewählter Aspekte von BVGE $2018 \mathrm{VI} / 5$

Sarah Frehner / Annina Mullis *

Im kommentierten Urteil hatte das Bundesverwaltungsgericht im Fall eines kurdischen Beschwerdeführers türkischer Herkunft über seine Asylunwürdigkeit zu entscheiden und setzte sich darin erstmals eingehend mit dem unbestimmten Rechtsbegriff der «Gefährdung der inneren und äusseren Sicherheit der Schweiz» nach Art. 53 lit. b AsylG auseinander. Im Ergebnis bleiben aber sowohl Kriterien als auch die Beweisanforderungen unscharf. Auffallend ist zudem die starke Gewichtung aussenpolitischer Aspekte. Insgesamt wirft das Urteil zahlreiche rechtliche und tatsächliche Fragen auf, so insbesondere in Bezug auf die extensive Auslegung von Art. 53 lit. b AsylG (III.1.), das Beweismass und die Verteilung der Beweislast (III.2.) und die Erkenntnisgrundlage (III.3.).

I. Sachverhalt 155

II. Kurzzusammenfassung Erwägungen BVGer ............................................ 155

III. Kommentierung ausgewählter Aspekte ................................................... 157

1. Gefährdung der inneren und äusseren Sicherheit nach Art. 53 lit. b AsylG .. 158

2. Beweismass und Beweislastumkehr ..................................................... 163

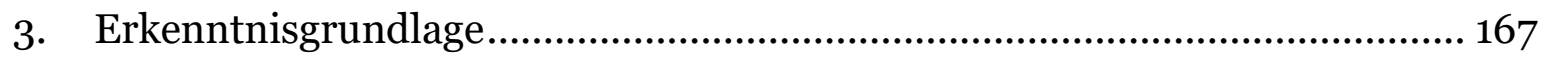

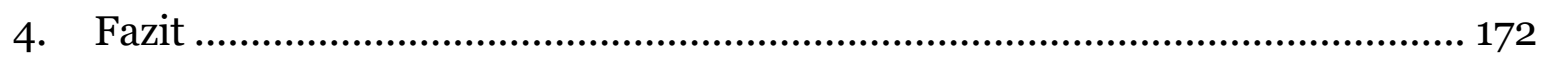

Zitiervorschlag: Sarah Frehner / Annina Mullis, Asylunwürdigkeit wegen «Gefährdung der inneren oder äusseren Sicherheit»: von abstrakter Gefährdung und Beweislastumkehr, in: sui-generis 2019, S. 154

URL: $\quad$ sui-generis.ch/100

DOI: $\quad$ https://doi.org/10.21257/sg.100

\footnotetext{
Sarah Frehner (sarah.frehner[at]fluechtlingshilfe.ch), MA European Studies, ist juristische Mitarbeiterin bei der Schweizerischen Flüchtlingshilfe (SFH), spezialisiert auf materielles Asylrecht und Menschenrechtsfragen, sie ist Mitglied der Demokratischen Jurist*innen Schweiz (DJS). Annina Mullis (mullis[at]advokatur4a.ch), MLaw, Rechtsanwältin in Bern, ist Vorstandsmitglied der DJS sowie der European Association of Lawyers for Democracy \& World Human Rights (ELDH) und nimmt seit 2012 als Delegierte der DJS sowie der ELDH regelmässig an Prozessbeobachtungen in der Türkei teil.
}

Dieses Werk ist lizenziert unter einer Creative Commons Namensnennung - Weitergabe unter gleichen Bedingungen 4.0 International Lizenz. 


\section{Sachverhalt}

1 Mit BVGE 2018 VI/5 vom 25. September $2018^{1}$ entschied das Bundesverwaltungsgericht (BVGer) über die Beschwerde eines Kurden türkischer Staatsangehörigkeit, der im April 2012 in der Schweiz ein Asylgesuch gestellt hatte. Nach Einreichen des Gesuchs war er im Dezember 2012 in den Niederlanden im Zusammenhang mit einer von ihm besuchten Veranstaltung neben weiteren Personen kurzzeitig festgenommen worden. Dieses Treffen soll nach Einschätzung der Schweizer Behörden von Komalen Ciwan (Gemeinschaft der Jugendlichen) und Yekîtiya Xwendekarên Kurdistan (Dachverband der Studierenden aus Kurdistan in Europa, $Y X K$ ) organisiert worden sein. Die Teilnahme des Beschwerdeführers am Kongress resp. die Festnahme hatten keine strafrechtlichen Konsequenzen; jedoch war der Betroffene dadurch in den Fokus des Nachrichtendienstes des Bundes (NDB) geraten. In einem Bericht an das Staatssekretariat für Migration $(\mathrm{SEM})^{2}$ hatte der NDB festgehalten, dass der Beschwerdeführer als Kadermitglied von Komalen Ciwan bekannt sei. Begründet wurde diese Annahme mit dem Hinweis auf eine öffentlich zugängliche Quelle, welche bestätige, dass die niederländische Polizei am 3. Dezember 2012 insgesamt 55 angeblich hochrangige Führungskräfte von Komalen Ciwan und der YXK verhaftet habe, wobei in Anbetracht seines Profils ausgeschlossen werden könne, dass der Beschwerdeführer Mitglied der $Y X K$ sei. Für den NDB sowie das SEM war damit

1 Vgl. Urteil des Bundesverwaltungsgerichts E2412/2014 (BVGE 2018 VI/5) vom 25. September 2018. Die Demokratischen Jurist*innen Schweiz (DJS) haben auf ihrer Homepage eine deutsche Übersetzung der Erwägungen veröffentlicht.

2 Damals noch Bundesamt für Migration (BFM). hinreichend nachgewiesen, dass er als Kadermitglied von Komalen Ciwan am Kongress teilgenommen habe. 3

2 Mit Entscheid vom 2. April 2014 anerkannte das SEM den Beschwerdeführer zwar als Flüchtling, schloss ihn jedoch gestützt auf die Einschätzung des NDB wegen Unwürdigkeit nach Art. 53 AsylG4 vom Asyl aus und verfügte die vorläufige Aufnahme5 in der Schweiz. Das SEM begründete den Entscheid mit der angeblichen Mitgliedschaft bei Ciwanên Azad (Freie Kurdische Jugend) - vom SEM als Nachfolgeorganisation von Komalen $\mathrm{Ci}$ wan bezeichnet ${ }^{6}$ - sowie seiner offenen Sympathiebekundungen für Abdullah Öcalan.7 Hiergegen erhob der Betroffene Beschwerde ans BVGer.

\section{Kurzzusammenfassung Erwägungen BVGer}

3 Gestützt auf Art. 53 AsylG wird Personen, die in der Schweiz als Flüchtling im Sinne der Genfer Flüchtlingskonvention $(\mathrm{GFK})^{8}$ anerkannt werden, dann kein Asyl gewährt9, wenn sie entweder wegen

3 Vgl. BVGE 2018 VI/5 Sachverhalt, insb. Bst. K und S. 4 Asylgesetz vom 26. Juni 1998, (AsylG; SR 142.31).

5 Der Beschwerdeführer erhält folglich anstelle einer Aufenthaltsbewilligung einen blossen FAusweis, diesbezüglich schreibt das BVGer von einem «statut moins favorable que l'asile», vgl. BVGE 2018 VI/5 E. 3.1.

6 Zum (angeblichen) Verhältnis zwischen Komalen Ciwan/Ciwanên Azad und der PKK siehe nachfolgende Ziffer 3.

7 Vgl. BVGE 2018 VI/5 Bst. L.

8 Abkommen über die Rechtsstellung der Flüchtlinge vom 28. Juli 1951, in Kraft getreten für die Schweiz am 21. April 1955, (SR 0.142.30).

9 Das BVGer hebt hervor, dass der Asylausschluss nach dem AsylG vom Ausschluss aus der Flüchtlingseigenschaft nach Art. 1 Abschnitt F GFK abzugrenzen ist. Diese Unterscheidung ist insbesondere für die Anwendbarkeit des flüchtlingsrechtlichen Gebots des Non-Refoulement von Bedeutung. Der Ausschluss von der Flüchtlingseigenschaft wird 
verwerflicher Handlungen des Asyls unwürdig sind (lit. a), die innere oder die äussere Sicherheit der Schweiz verletzt haben oder gefährden (lit. b) oder eine (militär)strafrechtliche Landesverweisung gegen sie ausgesprochen wurde (lit. c).

4 In BVGE $2018 \mathrm{VI} / 5$ prüft das BVGer einleitend die Voraussetzungen der Asylunwürdigkeit nach Art. 53 lit. a AsylG und stellt dabei fest, dass sich der Beschwerdeführer bisher nie strafbar gemacht hat. Er habe namentlich weder in der Türkei eine Handlung begangen, die nach Schweizer Recht als Verbrechen zu qualifizieren ist, noch seien in den Niederlanden oder in der Schweiz Strafverfahren gegen ihn eröffnet worden. Weiter gehöre er nicht zum bewaffneten Flügel der Partiya Karkerên Kurdistanê (Arbeiterpartei Kurdistans, $P K K$ ) und habe sich auch nicht an dessen kriminellen Handlungen beteiligt. Zwar nimmt das BVGer an, der Beschwerdeführer sei aktives Mitglied von Komalen Ciwan, ${ }^{10}$ einer Organisation, die insbesondere Kämpfer*innen sowie künftige Kader für die $P K K$ rekrutieren soll - eine individuelle (Mit-)Verantwortung an angeblichen Rekrutierungen wird dem Beschwerdeführer jedoch nicht vorgeworfen. Da somit keine «verwerflichen Handlungen» gegeben seien, könne der Asylausschluss nicht auf Art. 53 lit. a AsylG gestützt werden. ${ }^{11}$

Im Anschluss setzt sich das BVGer erstmals eingehend mit der Asylunwürdigkeit nach lit. b - und damit mit dem präventiven Asylausschluss aufgrund einer

vorliegend nicht in Betracht gezogen; vgl. BVGE $2018 \mathrm{VI} / 5$ E. 3.1.

10 Vgl. ebd. E. 5.1.

11 Vgl. ebd. E. 2.2, Schlussfolgerung in E. 2.2.3. angeblichen «Gefährdung der inneren oder äusseren Sicherheit der Schweiz» auseinander. ${ }^{12}$ Dem «PKK-nahen Mann» ${ }^{13}$, wie ihn das BVGer in seiner Medienmitteilung nennt, werden individuell weder die Mitgliedschaft in bzw. Unterstützung einer in der Schweiz verbotenen oder nach Art. 260 ${ }^{\text {ter }}$ StGB 14 als kriminell zu qualifizierenden Organisation vorgeworfen, noch soll er sich auf andere Weise strafbar gemacht haben. ${ }^{15}$ Das BVGer leitet von der Teilnahme des Beschwerdeführers am Kongress in den Niederlanden - im Gegensatz zur Vorinstanz und zum NDB - zwar keine Kaderstellung, aber dennoch seine aktive Mitgliedschaft bei Komalen Ciwan ab.16 Bezug nehmend auf ein Urteil des Bundesstrafgerichts (BStGer) aus dem Jahr $2012^{17}$ qualifiziert das BVGer Komalen Ciwan als Jugendorganisation der in der Schweiz nicht verbotenen $P K K$ und bezeichnet diese in seiner Medienmitteilung unmissverständlich als deren «geheime Unterorganisation» ${ }^{18}$. $\mathrm{Zu}$ den Hauptaufgaben von Komalen Ciwan gehöre die Rekrutierung von Kämpfer*innen für den bewaffneten Flügel der $P K K^{19}$ und in diesem Zusammenhang

12 Vgl. ebd. E. 4.4.

13 Asylgesuch eines $P K K$-nahen Mannes zu Recht abgewiesen, Medienmitteilung zum Urteil BVGE 2018 VI/5 vom 25. September 2018, St. Gallen 3. Oktober 2018.

14 Schweizerisches Strafgesetzbuch vom 21. Dezember 1937, (StGB; SR 311.0).

15 Vgl. BVGE $2018 \mathrm{VI} / 5$ E. 2.2.

16 Vgl. ebd. E. 5.1.

17 Vgl. TPF 2012 114; hier hatte die Beschwerdekammer des BStGer über die Auslieferung eines türkischen Staatsangehörigen nach Deutschland zu entscheiden. Die Beschwerde, namentlich die Einrede des politischen Delikts, wurde abgewiesen und die doppelte Strafbarkeit bejaht.

18 Vgl. Medienmitteilung zum Urteil BVGE 2018 VI/5 (Fn. 13).

19 Zum sogenannt «bewaffneten Flügel der $P K K$ » zählt das BVGer die Volksverteidigungskräfte (Hêzên Parastina Gel, HPG) sowie die Freiheits- 
auch die Organisation von Schulungen und «Ausbildungscamps».20

Der Beschwerdeführer hat die ihm zur Last gelegten Mitgliedschaften bei $\mathrm{Ci}$ wanên Azad und Komalen Ciwan gegenüber den Schweizer Behörden stets verneint und im Verfahren darüber hinaus angegeben, sich mit ausschliesslich friedlichen Mitteln für die «kurdische Sache» eingesetzt und sich vornehmlich an den Aktivitäten von kurdischen Kulturvereinen beteiligt zu haben. ${ }^{21}$ Als einziger Anhaltspunkt für eine gegenteilige Darstellung geht aus dem hier kommentierten Urteil der erwähnte Bericht des NDB und die darin festgehaltenen Annahmen hervor - dieser Hinweis ist aus Sicht des BVGer ausreichend. ${ }^{22}$ So hebt es im Urteil hervor, der eigentliche Nachweis einer die innere oder äussere Sicherheit möglicherweise verletzenden Handlung könne von den Behörden naturgemäss nur schwer erbracht werden, da (potenziell) gefährdendes Verhalten stets klandestin betrieben werde. Mit Blick auf diese grundsätzliche Beweis-

falken Kurdistans (Teyrênbazê Azadiya Kurdistan, TAK). Beide hat das BStGer als «kriminelle Organisationen» im Sinne von Art. 260 $\mathrm{O}^{\text {ter }}$ StGB qualifiziert; vgl. BVGE 2018 VI/5 E. 4.5 mit Hinweis auf TPF 2012114 E. 7.10.

20 Vgl. ebd.

21 Vgl. ebd. Bst. T.

22 Zur Würdigung nachrichtendienstlicher Informationen im Asylbeschwerdeverfahren vor dem BVGer siehe Bolz Susanne: Auch Dschihadisten haben (Verfahrens-)Rechte. Die Würdigung nachrichtendienstlicher Akten und Informationen in Asylbeschwerdeverfahren vor dem Bundesverwaltungsgericht, in: Jusletter vom 15. April 2019. Mit Blick auf den Anspruch auf rechtliches Gehör untersucht die Autorin im genannten Beitrag ein Merkblatt des NDB zum Procedere, wie Dokumente des NDB zu behandeln sind, die zu den Akten eines Verwaltungsverfahrens gehören. Das Merkblatt wurde am 9. September 2018 und damit vor dem kommentierten Urteil - herausgegeben und könne auf Anfrage beim NDB bezogen werden. problematik kehrt das BVGer die Beweislast um und führt aus, es obliege der gesuchstellenden Person, eine von den Behörden aufgestellte Tatsachenvermutung $\mathrm{zu}$ widerlegen. ${ }^{23}$ Diesbezüglich kommt das Gericht zum Schluss, der Beschwerdeführer habe sich nicht genügend von illegitimen Handlungen, die in der Folge vom Grund- und Menschenrecht auf freie Meinungsäusserung nicht mehr umfasst seien, distanziert. 24 Deshalb könne aus den Aktivitäten des Beschwerdeführers eine Gefährdung der inneren und äusseren Sicherheit der Schweiz abgeleitet werden, womit Art. 53 lit. b AsylG erfüllt sei.25

7 Im Ergebnis wird der Beschwerdeführer in der Schweiz zwar als Flüchtling im Sinne der GFK anerkannt, ihm wird jedoch kein Asyl gewährt. Als anerkannter Flüchtling kann sich der Beschwerdeführer auf das Gebot des Non-Refoulement ${ }^{26}$ berufen, weshalb sich der Wegweisungsvollzug als unzulässig erweist und er vorläufig in der Schweiz aufzunehmen ist. Die Beschwerde wird abgewiesen und der Entscheid des SEM bestätigt. ${ }^{27}$

\section{Kommentierung ausgewählter Aspekte}

8 Das Urteil des BVGer, welches von der Zeitschrift plädoyer in der Kategorie «Fehlurteil 2018» auf Rang zwei gewählt

23 Vgl. BVGE 2018 VI/5 E. 3.8.

24 Vgl. ebd. E. 3.7.5 und 5.6; zum geschützten Recht auf freie Meinungsäusserung gehörten eine pazifistische Verteidigung der Menschenrechte, jedoch nicht Aufrufe zu Gewalt oder Hass.

25 Vgl. ebd. E. $5.8 \mathrm{f}$.

26 Vgl. z.B. Schweizerische Flüchtlingshilfe (Hg.): Handbuch zum Asyl- und Wegweisungsverfahren, 2. Aufl., Bern 2015, S. 240 ff.

27 Vgl. BVGE 2018 VI/5 E. 8. 
wurde ${ }^{28}$, wirft in mehrfacher Hinsicht elementare Fragen auf: Rechtliche und tatsächliche Probleme bestehen insbesondere im Hinblick auf die weitreichende Auslegung des Asylausschlussgrundes nach Art. 53 lit. b AsylG (1.), das Beweismass und damit zusammenhängend die Gewichtung von geheimdienstlichen Einschätzungen sowie die Beweislastverteilung (2.), aber auch die Auswahl und Würdigung von Quellen zur Ermittlung des politischen Kontextes (3.). ${ }^{29}$

\section{Gefährdung der inneren und äusseren Sicherheit nach Art. 53 lit. b AsylG}

9 Um nach Art. 53 lit. b AsylG vom Asylstatus ausgeschlossen werden zu können, muss die betroffene Person nach dem Gesetzeswortlaut die innere oder die äussere Sicherheit der Schweiz entweder bereits verletzt haben oder gefährden wobei nachfolgend einzig die mögliche «Gefährdung» diskutiert wird.

Da sich das BVGer erstmals eingehend mit Art. 53 lit. b AsylG auseinandersetzt, hält es im besprochenen Urteil einleitend fest, die «innere und äussere Sicherheit» müssten als unbestimmte Rechtsbegriffe zunächst ausgelegt werden. Diese Auslegung könne jedoch nicht unabhängig von der weiteren Verwendung der Begriffe an

Schmid Gian Andrea: Fehlurteil 2018: «Prognose ohne Belege», in: plädoyer 1/19, S. $81 \mathrm{f}$.

29 Vgl. Frehner Sarah/Hruschka Constantin: Asylgewährung als moralischer Akt? BVGer (Schweiz), 25. September 2018, E-2412/2014, Asylausschluss gem. Art. 53 AsylG, in: $A S Y L$ 1/19, S. 45 ff.; als weiterer Aspekt wird hier zusätzlich die vom BVGer (nicht) vorgenommene Verhältnismässigkeitsprüfung diskutiert. anderer Stelle im AsylG3o oder etwa im AuG313 sowie im BüG32 erfolgen. 33

\section{a) Allgemeine Erwägungen}

11 Der eigentlichen Auslegung stellt das BVGer die Überlegung voran, dass die innere Sicherheit heute auch in einem internationalen Kontext verstanden werden müsse, weshalb es immer schwieriger werde, die «innere» und die «äussere» Sicherheit klar voneinander abzugrenzen; die innere und die äussere Sicherheit bezögen sich beide auf die Gewährleistung eines friedlichen Zusammenlebens - erstere auf nationaler und letztere auf internationaler Ebene. 34

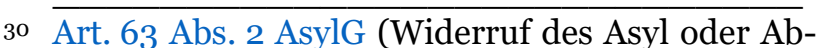
erkennung der Flüchtlingseigenschaft).

31 Bundesgesetz über die Ausländerinnen und Ausländer (AuG), seit 1. Januar 2019: Bundesgesetz über die Ausländerinnen und Ausländer und über die Integration vom 16. Dezember 2005, (AIG; SR 142.20): Art. 67 Abs. 4 (Einreiseverbot), Art. 68 Abs. 1 (Ausweisung) und Art. 103 Abs. 2 (Überwachung der Ankunft am Flughafen).

32 Bundesgesetz über das Schweizer Bürgerrecht vom 20. Juni 2014, (BüG; SR 141.09): Art. 11 lit. c (materielle Voraussetzungen ordentliche Einbürgerung), Art. 20 Abs. 2 (materielle Voraussetzungen erleichterte Einbürgerung) und Art. 26 Abs. 1 lit. e (Voraussetzungen für die Wiedereinbürgerung).

33 Vgl. BVGE 2018 VI/5 E. 3.2; das BVGer führt hier zudem aus, dass Art. 53 AsylG den Flüchtlingsstatus einer Peron nicht berühre. Unter Berücksichtigung seiner «beschränkten Auswirkung» auf den Flüchtlingsstatus seien die in Art. 53 AsylG enthaltenen Rechtsbegriffe mindestens ebenso streng auszulegen wie in den anderen Gesetzen, wenn nicht sogar noch strenger. Weiter hält das BVGer in E 3.3 fest, dass die «innere und äussere Sicherheit» vom Begriff der «öffentlichen Sicherheit und Ordnung» gemäss Art. 80 der Verordnung über Zulassung, Aufenthalt und Erwerbstätigkeit vom 24. Oktober 2007, (VZAE; SR 142.201) zu unterscheiden sei.

34 Vgl. ebd. E. 3.3. 
Mit Verweis auf die Praxis im Zusammenhang mit Art. 121 BV35 sowie auf Art. 3 BüV 36 bezeichnet das BVGer sowohl terroristische Aktivitäten als auch gewalttätigen Extremismus, verbotene nachrichtendienstliche Tätigkeiten und die organisierte Kriminalität als mögliche Gefährdungen für die Sicherheit der Schweiz. Gemäss Art. 3 BüV gefährde die Sicherheit, wer an Aktivitäten in den genannten Bereichen teilnehme, diese unterstütze, fördere oder dazu anwerbe.

\section{b) Neutralitäts- und aussenpolitische Erwägungen}

13 Darüber hinaus würden begrifflich auch Handlungen und Bestrebungen erfasst, welche die Beziehungen der Schweiz zu anderen Staaten ernsthaft gefährden könnten. 37 Diesbezüglich betont das BVGer insbesondere die schweizerische Neutralitätspolitik. Zur Wahrung der Sicherheitsinteressen bemühe sich die Schweiz aussenpolitisch um gute Beziehungen zu allen Staaten - unabhängig von ihrer politischen Organisationsform. ${ }^{8}$ In diesem Sinne hält das BVGer fest, die Sicherheit eines Landes hänge stark von der Qualität der Beziehungen zu anderen Staaten ab, weshalb sich auch migrationspolitische Fragen auf die diplomatischen Beziehungen zwischen einzelnen Staaten auswirken und so eine mögliche Gefährdung der «äusseren Sicherheit» der Schweiz begründen könnten. In solchen Konstellationen könne es gerechtfertigt erscheinen, den betreffenden Gesuchsteller*innen in der Schweiz

35 Bundesverfassung der Schweizerischen Eidgenossenschaft vom 18. April 1999, (BV; SR 101).

36 Verordnung über das Schweizer Bürgerrecht vom 17. Juni 2016, (BüV; SR 141.01).

37 Vgl. BVGE 2018 VI/5 E. $3.4 \mathrm{f}$.

38 Vgl. ebd. E. 3.6.2. keinen «so günstigen Rechtsstatus wie das Asyl» 39 zu gewähren.40

14 Da für die Annahme einer Gefährdung keine strafbare Handlung vorausgesetzt wird, könne nach Ansicht des BVGer für die Bejahung einer Bedrohung der äusseren Sicherheit 41 bereits die Zugehörigkeit $\mathrm{zu}$ einer Bewegung genügen, welche den radikalen Wandel des politischen Systems und der Machtverhältnisse im Herkunftsland oder einem Drittstaat fordere und implizit jeden Dialog ablehne. Dies sei auch dann der Fall, wenn die betroffene Person sich selber als «nicht gewaltbereit» bezeichne. Gemäss BVGer könne jede logistische Unterstützung einer terroristischen oder gewalttätigextremistischen Organisation - insbesondere Fundraising, Rekrutierung oder Propaganda - eine Bedrohung darstellen. Dies gelte selbst dann, wenn die betroffene Person kein formelles Mitglied der besagten Organisation sei. 42

\section{c) Strafrechtliche Erwägungen}

15 Anschliessend setzt sich das BVGer mit strafrechtlichen Aspekten auseinander: Strafbar sei namentlich die Beteiligung an und Unterstützung einer kriminellen Organisation nach Art. 260 ${ }^{\text {ter }}$ StGB. Weiter seien gewisse Organisationen gesetzlich verboten 43 und demnach jegliche Form

39 Ebd. E. 3.1.

40 Vgl. ebd. E. 3.6.

41 Mit Blick auf die innere Sicherheit hält das BVGer in E. 3.7.1 fest, dass dort eine Gefährdung angenommen werden könne, wo sich ethnische oder politische Konflikte vom Herkunftsstaat in die Schweiz verlagern könnten (Weiterführung von Konflikten in der Diaspora).

42 Vgl. ebd. E. 3.7.1.

43 Bundesgesetz vom 12. Dezember 2014 über das Verbot der Gruppierungen Al-Qaïda und Islamischer Staat sowie verwandter Organisationen vom 12. Dezember 2014, (SR 122); dieses komme gegenüber Art. 260 $0^{\text {ter }}$ StGB nur subsidiär zur An- 
von Beteiligung an diesen strafbar. Im Anwendungsbereich dieser Normen begründe die mit der Handlung bezweckte Förderung der kriminellen oder verbotenen Organisation die Strafbarkeit von ansonsten legalen Aktivitäten. 44

16 Für die Anwendbarkeit von Art. 53 lit.b AsylG sei es aber nicht vorausgesetzt, dass die Mitgliedschaft in einer Organisation an sich strafbar ist. Stehen in einem Fall Verbindungen zu Organisationen zur Diskussion, die weder als kriminell gelten, noch verboten sind, hänge die Bejahung einer Gefährdung der Sicherheit vom «Radikalitätsgrad» der Organisation sowie von der Art und dem Ausmass der betreffenden Handlungen ab. Mindestens müssten die individuellen Handlungen insoweit eine minimale potenzielle Bedrohung darstellen, um den NDB zu veranlassen, im Rahmen eines Konsultationsverfahrens dem SEM die Ablehnung eines Asylgesuchs zu empfehlen. 45

Betreffend die $P K K$ hält das BVGer fest, diese sei in der Schweiz weder verboten 46 noch stelle die Mitgliedschaft in der PKK als solche eine «verwerfliche Handlung» im Sinne von Art. 53 lit. a AsylG dar. 47

\section{d) Kommentar}

In BVGE 2018 VI/5 verzichtet das BVGer für die Bejahung der Asylunwürdigkeit auf das Vorliegen einer im In- oder Aus-

wendung. Allgemein: In der Schweiz kann der Bundesrat Organisationen gestützt auf Art. 185 BV und Art. 74 Bundesgesetz über den Nachrichtendienst vom 25. September 2015, (NDG; SR 121) (Revisionsverfahren eingeleitet) verbieten.

$44 \mathrm{Vgl}$. BVGE $2018 \mathrm{VI} / 5$ E. 3.7.3 f.

45 Vgl. ebd. E. 3.7.5.

46 Vgl. ebd. E. 3.7.4.

47 Vgl. ebd. E. 4.5. land begangenen Straftat. ${ }^{8}$ Im Gegensatz zur verwerflichen Handlung nach Art. 53 lit. a AsylG sowie zur Anordnung einer Landesverweisung gemäss lit.c, welche ein strafrechtlich vorwerfbares Verhalten resp. die Verurteilung wegen einer konkreten Straftat voraussetzen, hätten Massnahmen zur Wahrung der inneren und äusseren Sicherheit als Instrumente zum Schutz des Staats eine präventive Funktion.49 Durch die Entkoppelung der Asylunwürdigkeit von strafrechtlich relevantem Verhalten und mit einer geradezu ausufernden Auslegung des Begriffs der «Gefährdung» der inneren und äusseren Sicherheit konstruiert das BVGer aus Art. 53 lit. b AsylG einen Auffangtatbestand, dessen Konturen verschwommen bleiben. $5^{\circ}$ Die fehlende Trennschärfe beziehungsweise der daraus resultierende Spielraum der Behörden - lässt befürchten, dass die Beurteilung der (Nicht)Erfüllung der Voraussetzungen in $\mathrm{Zu}$ kunft eher von der politischen Grosswetterlage abhängig sein wird als von rechtlichen Überlegungen. Diesbezüglich besonders problematisch erscheint die vom BVGer postulierte «Staatspflicht», die Schweiz müsse sich um gute Beziehungen zu allen Staaten bemühen, unabhängig von ihrer politischen Organisationsform also auch zu autoritären Regimes und Diktaturen. Internationalen Beziehungen als Element zur Wahrung der Sicherheit der Schweiz wird damit derart viel Bedeutung beigemessen, dass deren Pflege sogar bei asylrechtlichen Entscheidungen

$48 \overline{\text { Zur bisherigen Praxis vgl. z.B. EMARK 2002/9 }}$ E. 7: Hier wird nicht zwischen den Alternativen in Art. 53 AsylG unterschieden, gleichzeitig aber das Prinzip betont, dass strafrechtlich vorwerfbares Verhalten einen Asylausschluss rechtfertigen könne.

49 Vgl. BVGE 2018 VI/5 E. 3.3.

50 Vgl. Frehner/Hruschka (Fn. 29), S. 45 f. 
berücksichtigt werden soll. Solche Überlegungen schwächen nichts weniger als das Fundament, auf welchem das Konzept des internationalen Schutzes als Ganzes aufbaut. Denn der Gedanke, Diplomatie in Asylentscheide einfliessen zu lassen, widerspricht der zwischenstaatlich allgemein anerkannten Vorstellung von Asylgewährung als Akt staatlicher Souveränität, der international nicht als feindliche Handlung gewertet werden darf. 51

Obwohl sich das Gericht mit dem Begriff der «Gefährdung der inneren und äusseren Sicherheit» befasst und für dessen Auslegung diverse Normen aus anderen Erlassen herangezogen hat, bleiben die konkreten Voraussetzungen letztendlich unklar. Strafrechtliche Kriterien wie Verbindungen zu kriminellen oder verbotenen Organisationen werden explizit verneint. Demgegenüber verlangt das BVGer Handlungen, die «eine minimale potenzielle Bedrohung» darstellen müssten. In diesem Zusammenhang bezeichnet auch Christof Riedo, Professor für Straf- und Strafprozessrecht an der Universität Freiburg, den Entscheid in der Zeitschrift plädoyer als «extrem stossend» $5^{2}$. Er vertritt die Ansicht, dass Gesuchsteller*innen ohne Vorliegen einer individuell vorwerfbaren, strafbaren Verhaltensweise keine konkrete Gefährdung der Sicherheit angelastet werden könne und betont, dass eine rein abstrakte Gefährdung für den Asylausschluss nicht ausreichen könne.53 Indem es das BVGer demgegenüber sogar genügen lassen will, dass nicht einmal die betroffene Person selbst direkt eine Bedrohung für das Land und seine Bewoh-

51 Vgl. Kälin Walter/Künzli Jörg: Universeller Menschenrechtsschutz, 4. Aufl., Basel 2019, S. 645, Rz. 18.70.

52 Schmid (Fn. 28), S. 81.

53 Vgl. ebd., S. 82. ner*innen darstellen müsse,54 entfernt sich das BVGer in rechtsstaatlich bedenklicher Weise vom Prinzip der Vorhersehbarkeit der auf ein bestimmtes Verhalten folgenden rechtlichen Konsequenzen.

20 An dieser Einschätzung ändert auch der vom BVGer gemachte Zusatz nichts, der NDB müsse sich- aus welchen Gründen auch immer - veranlasst sehen, dem SEM eine ablehnende Stellungnahme zukommen zu lassen. Im Gegenteil: Selbst wenn das SEM zusätzlich zur Einschätzung des NDB eine eigene Gefährdungsanalyse zu erstellen hat, wird mit dem Einbezug des Nachrichtendienstes einer sicherheitspolitischen Behörde im Asylverfahren eine potenziell gewichtige Rolle zugewiesen. Die in der vorliegenden Konstellation gegebene Überschneidung von asylrechtlichen Fragestellungen mit sicherheitspolitischen und strafrechtlichen Aspekten und deren Vermengung wirkt sich auch verfahrensrechtlich problematisch aus. Mit Blick auf den prozessualen Schutz der Betroffenen bestehen zwischen dem Asylund dem Strafverfahren grundsätzliche Unterschiede. Der Verurteilung wegen einer strafbaren Handlung ist im Strafprozess ein Verfahren mit expliziten Verteidigungsrechten 55 und einer umfassenden Beweisabnahme vorangestellt. Im Asylverfahren hingegen ist das Beweismass bereits insofern herabgesetzt, als dass für die Annahme eines Asylausschlussgrundes nicht zwingend eine strafrechtliche

\footnotetext{
Vgl. BVGE 2018 VI/5 E. 3.7.

55 Beschuldigte resp. ihre Rechtsvertretung haben Anspruch auf Einsicht in alle Verfahrensakten (Art. 101 und 108 der Schweizerischen Strafprozessordnung vom 5. Oktober 2007, [StPO]; SR 312.0), Beschuldigte müssen sich nicht selber belasten (insb. Art. 113 Abs. 1 StPO) und es gelten sowohl die Unschuldsvermutung als auch der Grundsatz in dubio pro reo (Art. 10 StPO).
} 
Verurteilung vorausgesetzt wird. $5^{6} \mathrm{Zudem}$ trifft die Gesuchsteller*innen im Asylverfahren eine Mitwirkungspflicht 57 und die Einsicht in einzelne Aktenstücke kann unter Einhaltung der entsprechenden Voraussetzungen für die gesamte Verfahrensdauer verweigert 58 werden. Dieser ohnehin bestehende Kontrast wird zusätzlich verstärkt, wenn nachrichtendienstliche Informationen und Quellen wesentlicher Bestandteil der Beweisführung sind - in Konstellationen wie der vorliegenden wird dies stets der Fall sein59 -, da der

56 Für die «verwaltungsrechtliche Annahme» strafrechtlich relevanter Handlungen in der Schweiz ist es erforderlich, dass im konkreten Fall eine Anklage erhoben wurde und entweder ein Geständnis oder eine liquide Beweislage vorliegt. Haben die Taten im Ausland stattgefunden, liegen häufig keine Strafurteile vor, weshalb bloss «ernsthafte Gründe» für die Annahme eines Ausschlussgrundes vorliegen müssen. Erforderlich sind substanziell verdichtete Verdachtsmomente, blosse Mutmassungen genügen nicht; vgl. Schweizerische Flüchtlingshilfe (Fn. 26), S. 215 f. und 227. Geht es um Straftaten, die nach der Flucht und in europäischen Nachbarstaaten verübt worden sein sollen, sind diese zwar im Ausland begangen, nach Ansicht der Autorinnen ist in dieser Konstellation jedoch das gleiche Beweismass wie bei Taten im Inland anzuwenden. Stehen - wie hier - z.B. in den Niederlanden begangene, potenziell strafrechtlich relevante Handlungen infrage, sind keine Beweisprobleme ersichtlich, welche die weitere Reduktion des Beweismasses rechtsfertigen würden.

57 Art. 8 AsylG.

58 Art. 6 AsylG i.V.m. Art. 26 ff. Bundesgesetz über das Verwaltungsverfahren vom 20. Dezember 1968, (VwVG; SR 172.021). Wird einer Partei die Einsichtnahme in Aktenstücke verweigert, darf auf diese gemäss Art. 28 VwVG zwar auch in einem öffentlich-rechtlichen Verfahren nur insoweit zum Nachteil der Partei abgestellt werden, wenn ihr die Behörde von dem für die Sache wesentlichen Inhalt mündlich oder schriftlich Kenntnis und ihr ausserdem Gelegenheit gegeben hat, sich zu äussern und Gegenbeweismittel zu bezeichnen. Dennoch ist anzumerken, dass ohne Kenntnis über die vollständigen Akten und insbesondere die «Metadaten» der Inhalte - wie z.B. die Quelle der Information - das Erbringen eines Gegenbeweises erheblich erschwert ist.

59 Eine Einschätzung des NDB ist gemäss BVGer Voraussetzung für die Anwendung von Art. 53
Rahmen für die Ausübung des Rechts auf Akteneinsicht mit Verweis auf spezifische Geheimhaltungs- resp. Sicherheitsinteressen weiter verengt wird.60 Es ist zu befürchten, dass betroffene Personen - begründet mit aus nachrichtendienstlicher Perspektive überwiegenden Sicherheitsinteressen $^{61}$ - auch vor dem BVGer keine (umfassende) Einsicht in die betreffenden Akten erhalten werden. ${ }^{62}$ Wird sodann

lit. b AsylG; vgl. BVGE 2018 VI/5 E. 3.8, für weitere Ausführungen siehe nachfolgende Ziffer 2.

6o Bolz ist dahingehend zuzustimmen, dass die am Verfahren beteiligten Behörden gehalten sind, die verwaltungsrechtlichen Vorgaben zum rechtlichen Gehör mit höchster Sorgfalt einzuhalten, sobald der Vorwurf von terroristischen oder staatsgefährdenden Aktivitäten im Raum steht genau diese Sorgfalt haben sowohl das SEM als auch das BVGer in diesem Fall jedoch vermissen lassen. Deshalb begrüssen die Autorinnen den von Bolz formulierten Vorschlag, eine rechtliche Grundlage für eine Spezialzuständigkeit des Bundesgerichts zu schaffen, sofern damit den Betroffenen in diesem für sie sensiblen Bereich der Zugang zu einer weiteren Rechtsmittelinstanz ermöglicht wird; zum Ganzen siehe Bolz (Fn. 22).

61 Im Vordergrund steht insbesondere die Regelung über den Quellenschutz für klassifizierte Akten und Informationen nach Art. 35 NDG; zum Interessenkonflikt zwischen Geheimhaltung und Anspruch auf rechtliches Gehör, welcher als Teilgehalt auch das Recht auf Akteneinsicht enthält, siehe Bolz (Fn. 22), Rz. 18-20.

62 Das BVGer schreibt unter Bst. S., das SEM habe den Beschwerdeführer nur ungenügend über den Inhalt des Berichts des NDB in Kenntnis gesetzt, weshalb das BVGer dies mit Verfügung vom 24. Oktober 2017 nachgeholt habe. Der Beschwerdeführer hatte somit erst im Herbst 2017 Zugang zu allen für das Verfahren wesentlichen Informationen erhalten (vgl. auch E. 5.5). Einsicht in den Amtsbericht resp. die Quellen des NDB und die übrigen dort über ihn gespeicherten Daten wurde ihm jedoch nicht gewährt. Weder aus dem Entscheid des SEM noch aus dem Urteil des BVGer geht hervor, ob es sich um klassifizierte Akten des NDB gehandelt hat. Die Tatsache, dass sich die Annahme, der Beschwerdeführer sei (Kader-)Mitglied von Komalen Ciwan, auf eine öffentlich zugängliche Quelle stützte (vgl. Bst. S), spricht vorliegend aber gegen das Zurückgreifen auf klassifizierte Akten oder schützenswerte Quellen, die dem Beschwerdeführer nicht hätten offen gelegt werden können. Zudem sind die Amtsberichte des NDB - hier die Zusammenfas- 
zusätzlich noch die Beweislast zu Ungunsten der Beschwerdeführer*innen umgekehrt ${ }^{63}$, führt dies im Vergleich zu einem strafrechtlichen Verfahren zu einer derart massiven prozessualen Schlechterstellung der Betroffenen, welche diese - auch wenn deren Flüchtlingseigenschaft nicht betroffen ist - nicht nur an empfindlicher Stelle trifft, sondern darüber hinaus die Gewährleistung eines fairen Verfahrens sowie die Waffengleichheit 64 in diesem spezifischen Ausschnitt des asylrechtlichen Verfahrens infrage stellt.

\section{Beweismass und}

\section{Beweislastumkehr}

Wie bereits ausgeführt, wird dem Beschwerdeführer vorliegend keinerlei strafrechtlich relevantes Verhalten vorgeworfen. Damit ist ausgeschlossen, dass in casu eine Verletzung der inneren oder

sung seiner Erkenntnisse zuhanden des SEM - in der Regel nicht klassifiziert, weshalb in diese grundsätzlich Einsicht gewährt werden kann (vgl. Bolz [Fn. 22], Rz. 25). Weshalb sich die Akteneinsicht vorliegend dennoch auf das blosse Zur-Kenntnis-Bringen der wesentlichen Informationen durch das BVGer beschränkte, ist erstens weder ersichtlich noch im Urteil begründet. Dies entspricht zweitens nicht dem von Bolz (Fn. 22) zur bestmöglichen Wahrung der Rechte der Betroffenen skizzierten Vorgehen bei der Verwertung nachrichtendienstlicher Informationen in einem Beschwerdeverfahren vor dem BVGer, wonach Amtsberichte und nachrichtendienstliche Zusammenfassungen klassifizierter Quellen grundsätzlich zum parteiöffentlichen Teil eines Dossiers gehören und einsehbar sind (Rz. 28-40, insb. 33). Mit Blick auf diese Vorgaben drängt sich die Frage auf, ob vorliegend das rechtliche Gehör des Beschwerdeführers verletzt wurde.

63 In E. 3.8 verlangt das BVGer konkrete Gegenbeweise oder das Glaubhaftmachen (des Gegenteils) durch fundierte, schlüssige und plausible Aussagen, um eine behördlich aufgestellte Tatsachenvermutung umstossen zu können; zum Ganzen siehe nachfolgende Ziffer 2.

64 Die Rechtsprechung leitet sowohl den allgemeinen Fairnessgrundsatz als auch die Waffengleichheit u.a. aus Art. 29 Abs. 1 BV ab; statt vieler vgl. BGE 139 I 121. äusseren Sicherheit bereits erfolgt ist; im Fokus der Prüfung stehen somit einzig die Voraussetzungen für die Bejahung einer Gefährdung sowie die beweisrechtlichen Voraussetzungen dafür.

\section{a) Allgemeine Erwägungen}

22

Laut BVGer genüge es, um eine Gefährdung der Sicherheit der Schweiz annehmen zu können, wenn auf der Basis konkreter Indizien ernsthafte Gründe vorlägen, welche die Annahme einer solchen Bedrohung rechtfertigten. ${ }^{65}$ Anzeichen und Indizien - also indirekte Beweise sollen deshalb als Begründungsgrundlage zugelassen werden, weil nach Ansicht des BVGer (potenziell) gefährdendes Verhalten stets im Untergrund betrieben werde und deshalb der eigentliche Nachweis einer Handlung, welche die innere oder äussere Sicherheit verletzen könnte, für die Behörden naturgemäss nur schwer zu erbringen sei. Blosse Mutmassungen würden jedoch nicht ausreichen, um die Vermutung gefährdender Handlungen zu begründen. Eine hinreichende Nähe zu Organisationen, die direkt oder indirekt zur Unterstützung oder Begehung von terroristischen Akten oder gewalttätigextremistischen Handlungen beitrügen vorliegend zur $P K K^{66}$ - soll jedoch genügen. ${ }^{67}$

65 Vgl. BVGE $2018 \mathrm{VI} / 5$ E. 3.7.

66 In den Erwägungen des BVGer wird die $P K K$ als Konstruktion verschiedener Gruppierungen und Unterorganisationen dargestellt, die über eine einheitliche und übergeordnete Entscheidungsstruktur verfüge. Die $P K K$ an sich ist in der Schweiz weder verboten, noch wurde diese bisher vom BStGer als kriminelle Organisation im Sinne von Art. $260^{\text {ter }}$ StGB eingestuft - einzig die $H P G$ und die TAK (siehe Fn. 19) wurden bisher nach dem StGB als kriminelle Organisationen qualifiziert; zur Auseinandersetzung mit der Struktur der $P K K$ siehe nachfolgende Ziffer 3.

67 Vgl. BVGE $2018 \mathrm{VI} / 5$ E. $3.8 \mathrm{f}$. 

BVGer die Konsultation des Nachrichtendienstes. Als zuständige Sicherheitsbehörde sei der NDB befugt und verpflichtet, dem SEM die für die Beurteilung benötigten (personenbezogenen) Informationen weiterzuleiten. Zwar hält das BVGer fest, die Einschätzungen des NDB seien im Asylverfahren nicht verbindlich. Dies aber nur um sogleich anzuführen, dass das SEM nicht ohne triftige Gründe davon abweichen dürfe. ${ }^{68}$ Dort, wo behördlich eine Gefährdung vermutet wird, soll zudem neu die Beweislast bei den Gesuchsteller*innen liegen. Konkret auferlegt das BVGer den betroffenen Personen die Pflicht, betreffend im Verfahren aufgestellte Tatsachenvermutungen den Gegenbeweis erbringen zu müssen. 69

Um eine solche behördliche Tatsachenvermutung umstossen zu können, verlangt das BVGer, dass gegenüber den Behörden relevante und unbekannte Tatsachen vorgebracht werden, oder - bei Mangel an konkreten Gegenbeweisen das Gegenteil durch fundierte, schlüssige und plausible Aussagen glaubhaft gemacht wird. ${ }^{70}$ Weiter bestehe eine potentielle Bedrohung dann nicht mehr, wenn sich die betroffene Person nach der Flucht in hinreichend erkennbarer und glaubhafter Art und Weise von der Ideologie, den Zielen und den Mitteln der fraglichen «radikalen Organisation» distanziert habe. Hierfür müsse jeglicher Kontakt mit der Organisation, ihren Deckorganisationen und deren rechtswidrigen Handlungen abgebrochen werden, wobei die Anforderungen umso strenger seien, je höher die Stellung der betroffenen Person

68 Zum Ganzen siehe Bolz (Fn. 22).

$69 \mathrm{Vgl}$. BVGE $2018 \mathrm{VI} / 5$ E. 3.8. und 3.10.

70 Vgl. ebd. E. 3.8. war, oder wenn es sich um eine besonders radikale Gruppe gehandelt habe. ${ }^{71}$

\section{b) Anwendung im konkreten Fall}

25 Vorliegend schreibt das BVGer dem Beschwerdeführer die aktive Mitgliedschaft bei Komalen Ciwan ${ }^{72}$ zu, einer Organisation, der nachgesagt wird, als $P K K$ Jugendorganisation insbesondere Kämpfer*innen sowie künftige Kader für die $P K K$ zu rekrutieren.73 Als einziger, diesbezüglicher Hinweis ist im Urteil die vorübergehende Festnahme des Beschwerdeführers in den Niederlanden erwähnt.

26 Das BVGer kommt zwar zum Schluss, der Beweis, dass der Beschwerdeführer eine Führungsrolle für Komalen Ciwan übernommen oder durch seine Handlungen die deliktischen Tätigkeiten des bewaffneten Flügels der $P K K$ begünstigt hätte, habe nicht erbracht werden können. 74 Dies sei jedoch auch gar nicht nötig, da bereits die blosse Zugehörigkeit zu dieser «Untergrundorganisation», welche die Unterstützung des bewaffneten Flügels der $P K K$ durch die Rekrutierung von Kämpfer*innen zum Ziel habe, genüge, um eine gefährdende Handlung nach Art. 53 lit. b AsylG anzunehmen.75 Das BVGer erkennt hier also eine Tatsachenvermutung im Sinne einer allgemeinen Erwägung, wel-

${ }_{71}$ Vgl. ebd. E. 3.11.

72 Zum (angeblichen) Verhältnis zwischen Komalen Ciwan/Ciwanên Azad und der PKK siehe nachfolgende Ziffer 3.

73 Vgl. BVGE 2018 VI/5 E. 2.2.

74 Demgegenüber hatte der NDB aus der Annahme, «gewöhnliche Mitglieder» würden zu solchen Kongressen nicht eingeladen, abgeleitet, dem Beschwerdeführer müsse innerhalb der Organisation eine leitende Stellung zukommen; vgl. ebd. E. 5.1.

75 Vgl. ebd. 
che der Betroffene im Verfahren widerlegen müsste.

Diesbezüglich führt das BVGer aus, der Beschwerdeführer habe sich auf ausweichende Erklärungen beschränkt, namentlich ohne Einzelheiten über das Treffen in den Niederlanden zu nennen. Im November 2017 habe der Beschwerdeführer im Rahmen einer schriftlichen Stellungnahme zwar seine Mitgliedschaft bei Ciwanên Azad - und damit implizit auch bei Komalen Ciwan - abgestritten, er habe aber nicht nur keine wesentlichen neuen Informationen offengelegt, sondern vielmehr versucht, über wesentliche Tatsachen einen falschen Eindruck zu erwecken. Damit habe er seine Mitwirkungspflicht zur Feststellung von Tatsachen, die er besser hätte kennen müssen als die Schweizer Behörden, nicht erfüllt und es müsse davon ausgegangen werden, dass er fallrelevante Tatsachen verschwiegen habe. 76

Auf die Vorbringen des Beschwerdeführers, er habe sich stets nur im Rahmen eines legitimen und friedlichen Engagements für die Achtung der Menschenrechte der kurdischen Bevölkerung in der Türkei politisch betätigt, geht das BVGer nicht ein. Mit Blick auf die Anzahl Teilnehmer*innen an pro-PKK-Demonstrationen in den Niederlanden oder in Deutschland, an welchen Tausende oder sogar Zehntausende teilnähmen, könne es sich nach Ansicht des BVGer bei der konkreten Veranstaltung in den Niederlanden nicht wie vom Beschwerdeführer behauptet um einen öffentlichen Kongress gehandelt haben. Das BVGer nimmt an, die Zahl der Besucher*innen hätte erheblich höher sein müssen, wenn die Teilnahme für alle

76 Vgl. ebd. E. 5.2 ff.
Sympathisant*innen offen gewesen wäre.77 Damit habe der Beschwerdeführer an Aktivitäten klandestiner Teile der $P K K$ Jugend-organisation teilgenommen. Da diese aufgrund der angeblichen Beteiligung an der Rekrutierung von zukünftigen $P K K$-Kadern sowie Kämpfer*innen für den bewaffneten Flügel der $P K K$ als Gefahr für die innere und äussere Sicherheit der Schweiz angesehen werden müssten, könne er sich nicht auf die Ausübung grund- und menschenrechtlich geschützter Aktivitäten berufen. 78

29 Abschliessend hält das BVGer fest, aus den vom NDB und vom SEM übermittelten Fakten gehe hervor, dass der Beschwerdeführer für Komalen Ciwan, deren Aktivitäten eine Bedrohung für die innere und äussere Sicherheit der Schweiz darstellten, 79 tätig gewesen sei. Im Rahmen seiner Äusserungen habe er seine Beteiligung lediglich abgestritten, aber keinerlei Reue gezeigt und sich weder klar noch in glaubwürdiger Weise distanziert. Damit habe er die Vermutung, er sei auch heute immer noch innerhalb der Organisation oder für diese aktiv, ${ }^{80}$ nicht widerlegen können. ${ }^{81}$

\footnotetext{
Vgl. ebd. E. 5.5.

Vgl. ebd. E. 4.7 und 5.6.

Vgl. ebd. E. 3.7.1 und 4.8.

80 Das BVGer hält sogar fest, es könne nicht ausgeschlossen werden, dass der Beschwerdeführer bisher «im Geheimen» in der Schweiz oder in Europa weitere Funktionen wahrgenommen habe, immer noch innehabe oder sogar in kurdische Gebiete habe zurückkehren können oder dies in Zukunft tun könnte, um seinen Führungsstatus durch die Teilnahme an Aktivitäten der PKK oder mit ihr verbundenen Gruppen auszubauen; vgl. ebd. E. 5.7.

81 Vgl. ebd. E. 5.8.
} 


\section{c) Kommentar}

30 Wie zuvor schon kritisiert, lassen die Ausführungen des BVGer insbesondere objektive und klar umrissene Kriterien für die Annahme einer von einer bestimmten Person konkret ausgehenden Gefährdung vermissen. Prozedural wird einzig die Konsultation des NDB vorausgesetzt, dessen Quellen und Grundlagen der Informationsbeschaffung für die Betroffenen und ihre Rechtsvertretungen jedoch nur beschränkt einsehbar sind ${ }^{82}$ und damit einer tatsächlichen Überprüfung durch die Verfahrenspartei nicht offenstehen. Zwar führt das BVGer aus, das SEM sei angehalten, unter Berücksichtigung der gesamten Umstände und der vollständigen Akten eine eigene Einschätzung des Gefahrenpotenzials zu erarbeiten. Gleichzeitig soll das SEM aber nicht ohne triftige Gründe von der Einschätzung des NDB abweichen, 83 womit die unmittelbar zuvor statuierte Pflicht zur eigenständigen (Über)Prüfung durch das SEM in der Praxis sogleich wieder eingeschränkt wird.

31 Besonders problematisch beurteilen die Autorinnen die mit der Umkehr der Beweislast vorgeschlagene erhebliche beweisrechtliche Verschiebung $\mathrm{zu}$ Lasten der Gesuchsteller*innen. Bereits vor BVGE 2018 VI/5 hatte die Praxis für den Ausschluss aus der Flüchtlingseigenschaft ein reduziertes Beweiserfordernis entwickelt, um der, betreffend allfällige Vorgänge in den Herkunftsstaaten, grundsätzlich erschwerten Beweislage Rechnung zu tragen. In diesem Sinne muss das Vorliegen eines Ausschlussgrundes nach der GFK behördlich nicht bewiesen wer-

$82 \overline{\text { Zur Einsichtnahme in Dokumente des NDB und }}$ zu deren Verwertung im Verwaltungsverfahren siehe Bolz (Fn. 22), Rz. 23-46.

83 Vgl. BVGE 2018 VI/5 (Fn.1) E. 3.10. den, sondern es müssen lediglich schwerwiegende Gründe vorliegen, die eine entsprechende Annahme rechtfertigen. ${ }^{84}$ Bis anhin galt dieser Massstab auch im Anwendungsbereich des Asylausschluss nach Art. 53 AsylG, wobei die Schwelle niedriger angesetzt werden durfte als beim Ausschluss aus der Flüchtlingseigenschaft. ${ }^{85}$ Die Beweislast lag jedoch beim SEM. 86

32 Nun wird vom Beschwerdeführer das Unmögliche gefordert: der Beweis für das Nichtbestehen klandestiner Tätigkeiten. ${ }^{87}$ Wie dieser Nachweis konkret hätte erbracht werden können, bleibt nach der Lektüre des Urteils offen. Vorliegend hätte der Beschwerdeführer zum einen keine Reue gezeigt und sich zum anderen nicht glaubwürdig distanziert. ${ }^{88}$ Weitere Ausführungen zum im Verwaltungsverfahren unangebrachten «Erfordernis der Reue» 89 macht das BVGer nicht. Als konkretisierender Hinweis lässt sich den Erwägungen aber entnehmen, dass betroffene Personen den Kontakt zu den fraglichen Organisationen ganz abgebrochen haben müssten. Zur Beurteilung könnten etwa die Umstände, die Ernsthaftigkeit, die Stabilität sowie die Dauer der Distanzierung oder gar eine eventuelle «Deradikalisierung» herangezogen werden. ${ }^{\circ 0}$ Humanrights.ch benennt das sich hieraus für eine*n Gesuchsteller*in im Asylverfahren ergebende Paradox: Einerseits muss - etwa aufgrund politischer Betätigung - eine asylrelevante Verfolgung im

84 Vgl. z.B. BVGE 2013/36.

85 Vgl. EMARK 2009/2 E. 7.

86 Vgl. BVGE 2013/23 E. $3.3 \mathrm{f}$.

87 Vgl. Frehner/Hruschka (Fn. 29), S. 46.

88 Vgl. BVGE 2018 VI/5 (Fn.1) E. 5.8.

89 Zur Vermischung strafrechtlicher Begrifflichkeiten mit verwaltungsrechtlichen Prüfungsmassstäben siehe Frehner/Hruschka (Fn. 29), S. 46.

90 Vgl. BVGE 2018 VI/5 (Fn.1) E. 3.11. 
Herkunftsstaat nachgewiesen werden. Andererseits soll sich die betroffene Person von den mit dem politischen Aktivismus im Herkunftsstaat verbundenen Organisationen glaubhaft und vollständig distanzieren..$^{1}$

Auffallend ist zudem, wie stark sich das BVGer auf Mutmassungen stützt, die den Ausschluss eigentlich gerade nicht rechtfertigen können sollten:92 Es könne z.B. nicht ausgeschlossen werden, dass sich der Beschwerdeführer auch über die bekannte Teilnahme am genannten Kongress hinaus - ohne entsprechende Kenntnis der Behörden - in der Schweiz oder in Europa aktiv betätigt und in diesem Rahmen (weitere) Funktionen wahrgenommen habe. Es könne zudem auch nicht ausgeschlossen werden, dass er in kurdische Gebiete habe zurückkehren können oder dies in Zukunft tun könnte, um seinen Führungsstatus - der gemäss BVGer eben nicht bewiesen werden konnte - durch die Teilnahme an Aktivitäten der $P K K$ oder mit ihr verbundenen Gruppen auszubauen.93 Mit diesen Ausführungen scheint das Gericht das Beweismass noch weiter zu senken und schliesst den Beschwerdeführer im Ergebnis gestützt auf weitgehend weder bewiesene noch erhärtete Tatsachenbehauptungen vom Asyl aus. Die vom BVGer als Voraussetzung bezeichnete minimale potenzielle Bedrohung 94 wird damit zum breit angelegten Raster, welches - je nach politischer Kon-

$91 \overline{\text { Vgl. Bundesverwaltungsgericht stellt bei Asylaus- }}$ schluss die Unschuldsvermutung in Frage, Onlinebeitrag des Informationsportals humanrights.ch vom 5. Dezember 2018.

92 Vgl. BVGE $2018 \mathrm{VI} / 5$ (Fn. 1) E. 3.9.; zur bisherigen Praxis vgl. z.B. BVGE 2013/23 E. 3.3 und EMARK 2006/29 E. 4.2.

93 Vgl. BVGE 2018 VI/5 (Fn.1) E. 5.7.

94 Vgl. ebd. E. 3.7.5. junktur - im Einzelfall weiter oder enger gefasst werden kann.

\section{Erkenntnisgrundlage}

34 Gestützt auf Quellen verschiedener Art setzt sich das BVGer in BVGE $2018 \mathrm{VI} / 5$ mit dem Charakter, den Zielen sowie den Strukturen der $P K K$ auseinander und untersucht die Sicherheitsprobleme für die Schweiz resp. Europa, die durch die Präsenz von mit der $P K K$ verbundenen Gruppierungen auftreten können. An dieser Stelle soll die Auswahl sowie die Art und Qualität der verwendeten Quellen genauer betrachtet und diese öffentlich zugänglichen Informationen gegenübergestellt werden.

\section{a) Allgemeine Erwägungen}

35 Wie bereits festgestellt ist die $P K K$ in der Schweiz als Organisation nicht verboten. 95 Das BVGer verweist jedoch auf den Umstand, dass die $P K K$ auf der von der Europäischen Union (EU) erlassenen Liste der «terroristischen Bewegungen»96 erfasst sei, die - als EU-Liste - in der Schweiz zwar im Strafrecht keine Bedeutung habe, in verwaltungsrechtlichen Belangen aber trotzdem zu beachten sei. Die $P K K$ sei auf der Basis objektiv fundierter Indizien auf die erwähnte Liste der EU gesetzt worden, weshalb nicht ausgeschlossen werden könne, dass diese Organisation nicht bereits heute auch auf der nach Art. 72 Abs. 2 NDG vertraulich geführten Obser-

95 Vgl. ebd. E. 3.7.4.

96 Vgl. Durchführungsverordnung (EU) 2017/1420 des Rates vom 4. August 2017 zur Durchführung des Artikels 2 Absatz 3 der Verordnung (EG) Nr. 2580/2001 des Rates vom 27. Dezember 2001 über spezifische, gegen bestimmte Personen und Organisationen gerichtete restriktive Massnahmen zur Bekämpfung des Terrorismus und zur Aufhebung der Durchführungsverordnung (EU) 2017/150 des Rates vom 27. Januar 2017. 
vationsliste des NDB erfasst sei oder in Zukunft darin aufgenommen werden könnte. 97

Weiter habe das BStGer die $P K K$ an sich bisher nicht als kriminelle Organisation im Sinne von Art. 260 ter StGB eingestuft das gleiche gilt für Komalen Ciwan. Einzig die $H P G$ und die $T A K$ wurden bisher nach dem StGB als kriminelle Organisationen qualifiziert. 98

37 Die Analyse der Struktur der Organisationen übernimmt das BVGer aus einem Urteil des BStGer aus dem Jahr $2012^{99}$ welches sich wiederum auf ein Urteil des deutschen Bundesgerichtshofs aus dem Jahr 2010 bezieht - und gibt die darin enthaltenen Feststellungen wieder. Das zitierte Urteil des BStGer hält im Allgemeinen fest, die «Sektionen der $P K K$ » funktionierten klandestin, wechselten häufig ihre Bezeichnung und würden mit dem militärischen Arm zusammenarbeiten. Die Organisation sei straff und zentralisiert geführt, marxistisch-leninistisch geprägt und habe die Absicht, einen kurdischen sozialistisch-revolutionären Staat zu gründen. Hierfür werde Gewaltanwendung als legitimes Mittel erachtet. Anschliessend beschreibt das BVGer ein Geflecht verschiedener - angeblich von der $P K K$ gegründeter - Gremien und Organe, welche in der Türkei aber auch in Europa aktiv seien. Der Rechtsprechung des deutschen Bundesgerichtshofs folgend, hält das BVGer zudem fest, dass die $P K K$-Strukturen in Europa insgesamt weder organisatorisch noch in Bezug auf die Bildung ihres inneren Willens als von der Organisation in der Türkei unabhängig

\footnotetext{
97 Vgl. BVGE 2018 VI/5 (Fn.1) E. 4.3.

98 Vgl. ebd. E. 4.5 mit Verweis auf TPF 2012114 E. 7.9.3 und 7.10.

99 Vgl. TPF 2012114.
}

angesehen werden könnten. Hierzu gehöre auch Komalen Ciwan, die als eine der Jugendorganisationen die hauptsächliche Aufgabe habe, junge Kurd*innen für Kaderfunktionen oder als HPGKämpfer*innen zu rekrutieren. $\mathrm{Zu}$ diesem Zweck organisiere sie insbesondere «Ausbildungscamps». ${ }^{100}$ Daneben sei - gestützt auf den deutschen Verfassungsschutzbericht 2016 ${ }^{101}$ - im April 2013 die Freie Kurdische Jugend (Ciwanên Azad) als weitere Jugendorganisation gegründet worden. Beide Organisationen sollen jedoch denselben Personenkreis umfassen, ${ }^{102}$ weshalb sie das BVGer im Verhältnis zueinander auch als «Doubles» bezeichnet.103 Der Name Ciwanên Azad sei lediglich die offizielle Bezeichnung, die bei friedlichen Demonstrationen verwendet werde, während der Name Komalen Ciwan im Zusammenhang mit in der Öffentlichkeit negativ konnotierten Aktionen genutzt werde. ${ }^{104}$

38 Mit Blick auf die konkrete Situation in der Schweiz stellt das BVGer mit Verweis auf den Lagebericht des NDB für das Jahr $2018^{105}$ fest, dass die $P K K$ über ein hohes Mobilisierungspotential verfüge. So könne die $P K K$ schnell in ganz Europa Sympathisant*innen für Demonstrationen und Protestaktionen gegen Ereignisse in den kurdischen Gebieten der Türkei mobilisieren. Als bedeutendste Bedrohung in der Schweiz müssten mögliche Auseinandersetzungen zwischen PKK-

\footnotetext{
100 Vgl. BVGE 2018 VI/5 (Fn.1) E. 4.5.

101 Vgl. Verfassungsschutzbericht 2016, Deutschland, Bundesministerium des Innern, veröffentlicht Juni 2017.

102 Vgl. BVGE 2018 VI/5 (Fn.1) E. 4.7.

103 Vgl. ebd. E. 5.1.

104 Vgl. ebd. E. 4.7 mit Verweis auf den deutschen Verfassungsschutzbericht 2016 (Fn. 101), S. 243.

105 Vgl. Sicherheit Schweiz, Lagebericht 2018 des Nachrichtendienstes des Bundes, S. 42.
} 
Aktivist*innen und Anhänger*innen Erdoğans betrachtet werden, wobei eingeräumt wird, dass bisherige Demonstrationen und Proteste überwiegend friedlich verlaufen sind. Aber auch das Interesse des linksextremen Milieus an «Rojava», dem syrischen Teil Kurdistans, könne einen Risikofaktor für die Sicherheit der Schweiz darstellen. ${ }^{106}$

\section{b) Kommentar}

39 Auf den ersten Blick stützt das BVGer seinen Entscheid auf eine Vielzahl verschiedener Quellen ab - auf EU-Recht, auf die Rechtsprechung verschiedener Gerichte, auf nachrichtendienstliche Berichte. Auf den zweiten Blick zeigt sich jedoch schnell eine unsorgfältige und einseitige Ermittlung der kontexuellen Entscheidungsgrundlage.

40 Erstens verweist das BVGer auf die EUListe der terroristischen Bewegungen, erklärt diese für verwaltungsrechtlich beachtlich und widerspricht damit der eigenen Handhabung in früheren Urteilen, wo der EU-Liste keine Bedeutung beigemessen worden war. ${ }^{107}$ Die Berücksichtigung der EU-Liste rechtfertigt das BVGer insbesondere damit, dass die dortige Erfassung der $P K K$ auf der Basis objektiv fundierter Indizien erfolgt sei. Eben diese Annahme wurde kurz nach dem Urteilsspruch des BVGer von der zuständigen Gerichtsinstanz unmissverständlich widerlegt: So hat der Gerichtshof der Europäischen Union (EuGH) im November 2018 in einem Urteil festgestellt, die $P K K$ sei zwischen 2014 und 2017 - also während einer vorliegend re-

106 Vgl. BVGE 2018 VI/5 (Fn.1) E. 4.7 f.

107 Vgl. z.B. BVGE 2013/34 E. 7.2. levanten Periode - zu Unrecht auf dieser Liste geführt worden. ${ }^{108}$

41 Zweitens stützt sich das BVGer betreffend die Analyse der Struktur der PKK und der mit ihr affiliierten Organisationen vornehmlich auf ein Urteil des BStGer aus dem Jahr 2012. Darin verwertete das BStGer für die - vom BVGer übernommenen - Ausführungen, abgesehen von zwei unwesentlichen Ausnahmen, lediglich die Angaben aus einem Urteil des deutschen Bundesgerichtshofs vom 28. Oktober 2010; im Urteil des BStGer finden sich weder eine klare Referenz zum besagten Urteil109 noch Hinweise auf (andere) Primärquellen. ${ }^{110}$ Es fehlen damit nicht nur zuverlässige Quellenangaben, womit die Ausführungen nur schwer verifizierbar sind, sondern das BVGer stützt sich im kommentierten Urteil faktisch auf eine heute ohnehin veraltete Informationslage. Dies erscheint klar unzureichend.

42 Drittens zieht das BVGer verschiedene Berichte des deutschen und des Schweizer Nachrichtendienstes sowie von Europol heran - alle präsentieren in ihren zitierten Berichten praktisch keine Quellenangaben. Für eine tatsächlich vertiefte Analyse des politischen Kontextes - insbesondere in der vorliegenden Konstellation ein eigentlich zentrales Element der Urteilsbegründung - wäre der Beizug von umfassenden Informationen aus verlässli-

108 Vgl. Urteil des EuGH ECLI:EU:T:2018:788 vom 15. November 2018, (PKK gg. EU-Rat, RS T-316/14).

109 Die Autorinnen nehmen an, dass es sich beim zitierten Urteil um das Urteil des Bundesgerichtshof 3 StR 179/10 vom 28. Oktober 2010 handelt. Interessanterweise werden auch darin keinerlei Quellen angegeben. Vielmehr verweist der deutsche Bundesgerichtshof - ohne entsprechende Referenzen - auf die Feststellungen und Wertungen im angefochtenen Entscheid des Oberlandesgerichts (Vorinstanz).

110 Vgl. TPF 2012 114, insbesondere E. 7.6. 
chen und aktuellen Quellen notwendig gewesen. Die einzige nicht-staatliche Quelle, welche im Urteil aufgeführt wird, ist ein Artikel eines Richters des BVGer aus dem Jahr 2015; ${ }^{111}$ Einschätzungen von ausgewiesenen Expert*innen werden vergeblich gesucht.

43 In den Erwägungen des BVGer wird die $P K K$ als Konstruktion verschiedener Gruppierungen und Unterorganisationen dargestellt, die über eine einheitliche und übergeordnete Entscheidungsstruktur verfüge. Insgesamt wird das Bild einer zusammenhängenden, hierarchisch strukturierten Gesamtorganisation gezeichnet. Diese Darstellung der PKK sei jedoch in mehrfacher Hinsicht fehlerhaft, sagte der Wissenschaftler und Autor Kerem Schamberger ${ }^{112}$ gegenüber den Autorinnen. Zum einen könne nicht das ganze Spektrum der kurdischen Strukturen und Organisationen alleine der $P K K$ zu- resp. untergeordnet werden. Vielmehr bestünden verschiedene Gruppen und Initiativen nebeneinander, welche zwar sowohl die politischen Positionen von Abdullah Öcalan als gemeinsamen Bezugsrahmen teilten, als auch projektbezogen zusammenarbeiteten, zueinander aber in einem unabhängigen und insbesondere nicht weisungsgebundenen Verhältnis stünden. Mit Blick auf den konkreten Fall führte er aus, Jugendorganisationen verfolgten

111 Vgl. Haefeli Fulvio/Winter Gert: Gewaltanwendung und Asylunwürdigkeit am Beispiel der PKK, in: ZAR 2015, S. 97-104.

112 Kerem Schamberger ist Kommunikationswissenschaftler an der Ludwig-Maximilians-Universität in München. Zusammen mit Prof. Michael Meyen hat er das im September 2018 im Westend Verlag erschienene Buch «Die Kurden - ein Volk zwischen Unterdrückung und Rebellion» geschrieben. Die Autorinnen haben ihm das übersetze Urteil vorgelegt (vgl. Fn. 1) und danach am 29. Januar 2019 ein telefonisches Interview mit ihm geführt. nicht den Zweck der Rekrutierung, sondern hätten sich die politische Bildung als primäre Aufgabe gegeben. So sei die $Y X K$, welche den angeblich klandestinen Kongress in den Niederlanden mitorganisiert haben soll, an zahlreichen deutschen Universitäten als offizielle Student*innenorganisation registriert und anerkannt. Aber auch die Zahl der Besucher*innen spreche - wie vom Beschwerdeführer vorgebracht - nicht gegen die Öffentlichkeit der Veranstaltung. Auch wenn in Europa tatsächlich teilweise Grossdemonstrationen mit mehreren tausend Teilnehmer*innen stattfänden, sei dies keine Grundlage dafür auszuschliessen, dass insbesondere politische Informationsveranstaltungen und inhaltliche Seminare zwar öffentlich, aber in kleinerem Rahmen durchgeführt würden.

44 Zum anderen betonte Schamberger, das BVGer vernachlässige den innerhalb der $P K K$ vollzogenen - und von dieser selbst so bezeichneten - «Paradigmenwechsel».113 Die vom BVGer gewählte Beschreibung der $P K K$ als «marxistischleninistisch» mag bis in die 1990er-Jahre Geltung gehabt haben; mittlerweile habe sich die $P K K$ jedoch von der Idee der Schaffung eines unabhängigen Kurdistans als sozialistischen Staat gelöst und verfolge heute das Ziel föderaler Selbstverwaltung114 - dies explizit auch innerhalb der türkischen Staatsgrenzen. ${ }^{115}$ Dennoch ist

113 Vgl. Jongerden Joost: Gleichstellung der Geschlechter und radikale Demokratie, in: Brauns Nikolaus/Çakırö Murat (Hg.): Partisanen einer neuen Welt - Eine Geschichte der Linken und Arbeiterbewegung in der Türkei, 5. Aufl., Berlin 2019, S. 467 und $476 \mathrm{ff}$.

114 Vgl. ebd., S. 477.

115 Bereits 1993 habe Abdullah Öcalan im Rahmen einer Medienkonferenz erklärt, der politische Kampf sei auf Demokratie und die Respektierung der Menschenrechte gerichtet; die Kurd*innen 
zutreffend, dass es in jüngster Vergangenheit, insbesondere nach dem ersten Wahlerfolg der Halklarm Demokratik Partisi (Demokratische Partei der Völker, HDP) und ihrem Einzug ins nationale Parlament im Sommer 2015 sowie nach Abbruch der seit 2013 mit der PKK geführten Friedensgesprächen im Südosten der Türkei wieder zu gewaltsamen Auseinandersetzungen gekommen ist. ${ }^{116}$ Diesbezüglich entschied jedoch ein belgisches Gericht, es handle sich bei den Auseinandersetzungen in der Türkei nicht um Terrorismus, sondern um einen innerstaatlichen bewaffneten Konflikt. ${ }^{117}$ Auch wenn dieses Gerichtsurteil und damit die darin enthaltene Beurteilung nach heutigem Kenntnisstand noch nicht rechtskräftig zu sein scheint, macht dieser Entscheid aus Belgien deutlich, dass in dieser Frage Interpretationsspielraum besteht.

45 Das BVGer macht jedoch nicht nur von dem bestehenden Spielraum keinen Ge-

wollten sich nicht von der Türkei abspalten; vgl. van Gent Amalia: Leben auf Bruchlinien - Die Türkei auf der Suche nach sich selbst, Zürich 2008, S. 207. Spätestens um die Jahrtausendwende wurde innerhalb der $P K K$ endgültig die Frage diskutiert, ob die Unabhängigkeit tatsächlich in Form der Staatsgründung angestrebt werden soll und in der Folge die Selbstbestimmung vom Prinzip der Staatsgründung gelöst; vgl. Joost (Fn. 113), S. 467.

116 Als durch die verhältnismässig starke Vertretung der HDP im Parlament (im Juni 2015 hatte sie 80 von 550 Sitzen erhalten) die Alleinregierung der AKP nicht mehr gewährleistet schien, kündigte Erdoğan vorgezogene Neuwahlen an. Im Anschluss kam es u.a. zu pogromartigen Übergriffen auf HDP-Parteibüros, die seit 2013 geführten Friedensverhandlungen wurden beendet und das Militär startete in den überwiegend kurdischen Gebieten des Landes eine neue Offensive; vgl. Bozay Kemal/Kaygisız Hasan: Der neue Sultan Die Türkei zwischen Repression und Widerstand, Köln 2017, S. 102 f.

117 Vgl. ebd., S. 105; Azadî Maxime: Interview mit dem belgischen Rechtsanwalt Jan Fermon, in: Kurdistan Report Nr. 203, Mai/Juni 2019, S. 40 ff. brauch, sondern übernimmt faktisch das argumentative Narrativ der türkischen Regierung, welche die ganze Bandbreite der politischen Opposition unter dem Vorwand der Verbindungen zu einer aus Sicht der $A K P$-Regierung terroristischen Organisation - sei es die $P K K$ oder die Gülen-Bewegung - (strafrechtlich) verfolgt. ${ }^{118}$ In diesem Zusammenhang fällt auf, dass das BVGer im Urteil pauschal von der $P K K$ und ihren affiliierten Organisationen spricht, ohne zu präzisieren, worauf es sich in den jeweiligen Erwägungen genau bezieht oder stützt. ${ }^{119} \mathrm{Zu}-$ dem wird - abgesehen von der «Klandestinität» - nicht klar spezifiziert, welche Eigenschaften eine «PKK-nahe Organisation» aufweisen müsse, damit eine «hinreichende Nähe» zu dieser zur Asylunwürdigkeit führt.

46 Zusammenfassend erscheint das Informationsfundament des hier kommentierten Urteils mangelhaft. Für Herkunftsländerinformationen, die als Grundlage für die Prüfung der Flüchtlingseigenschaft von Gesuchsteller*innen dienen, gibt es klare Qualitätsstandards, welche die Anforderungen an die Ausgewogenheit, die Vielseitigkeit und die Verlässlichkeit von Quellen vorgeben. Nach den sowohl von der Schweizer ${ }^{120}$ als auch in der internationalen $^{121}$ Rechtsprechung anerkannten

118 Vgl. $\quad$ z.B. Kayserilioğlu Alp/Mullis Annina: Politische Justiz in der Türkei - Eine Einordnung der KCK-Verfahren, in: Forum Recht 04/16, S. 137 ff.; oder Alp Tarkan: «End of the State of Emergency - Old wine in new bottles?»: New Anti-Terror Law Gives the Power to Dismiss Any Judge, in: Stadelmann Thomas (Hg.): Democracy falling apart - Role and Function of Judicial Independence, Separation of Powers and the Rule of Law in a Constitutional Democracy, Bern 2018, S. 152.

119 Vgl. Frehner/Hruschka (Fn. 29), S. 47.

120 Vgl. z.B. BVGE 2015/10 E. 5.2.2.2.

121 Vgl. z.B. Urteil des EGMR 25904/07 vom 17. Juli 
Standards müssen sich die verwendeten Quellen insbesondere durch Unabhängigkeit, Zuverlässigkeit und Objektivität auszeichnen. Es ist nicht nachvollziehbar, weshalb diese Richtlinien nicht auch im Zusammenhang mit dem Asylausschluss zur Anwendung kommen sollten. Mindestens müsste aber in die Beweiswürdigung miteinfliessen, woher eine Information kommt, in welchen institutionellen oder politischen Kontext diese zu verorten und wie diese zeitlich/historisch einzuordnen ist.

Zwar ist das Gericht an keine starren Beweisregeln gebunden und es gibt keine Vorschriften im eigentlichen Sinne, welche einer Behörde "genau vorschreiben, wie ein gültiger Beweis zustande kommt und welchen Beweiswert die einzelnen Beweismittel im Verhältnis zueinander haben. Freie Beweiswürdigung ist aber nicht mit freiem Ermessen zu verwechseln»122. Vor dem Hintergrund der ohnehin dünnen Beweislage und der dem Beschwerdeführer überbürdeten Beweislast erscheint hier die unsorgfältig und einseitig - zu Ungunsten des Betroffenen vorgenommene Ermittlung der allgemeinen Faktenlage ausserhalb des zulässigen Rahmens im soeben zitierten Sinne.

\section{Fazit}

48 Spätestens mit BVGE $2018 \mathrm{VI} / 5$ ist die in sicherheitspolitischen Fragen präventive Neuausrichtung der Rechtsordnung ${ }^{123}$ auch im Asylrecht angekommen. Dies

2008 (N.A. gg. Vereinigtes Königreich), § 119-121.

122 BVGE 2013/34 E. 6.2.

123 Vgl. z.B. Achermann Jonas: Strafbarkeit von Versuch und Teilnahme bei Vorbereitungsdelikten, Dissertation, Zürich 2018; sicherheitspolitisch hat der Bundesrat angekündigt, das strafrechtliche Instrumentarium zur Verhütung von Terrorismus zu verstärken. wird vorliegend besonders am Umstand deutlich, dass das Gericht zwar festhält, die für die Schweiz im Zusammenhang mit der $P K K$ grösste Bedrohung der Sicherheit, beschränke sich auf mögliche Zusammenstösse zwischen PKKAktivist*innen und türkischen Nationalist*innen - obwohl diese bisher weitestgehend ausgeblieben seien. Trotzdem wird mit Blick auf den Beschwerdeführer gestützt auf weitgehend nicht belegte Verbindungen zu einer angeblich mit der $P K K$ affiliierten Gruppe eine von ihm ausgehende Gefährdung der inneren und äusseren Sicherheit der Schweiz bejaht, wobei das BVGer in den diesbezüglichen Erwägungen insbesondere aussen- und neutralitätspolitisch argumentiert. Mit der weiten Auslegung des Begriff der «inneren und äusseren Sicherheit der Schweiz», mit den an die gerechtfertigte Annahme einer möglichen Gefährdung tief gesetzten Anforderungen und nicht zuletzt mit der Umkehr der Beweislast wird einer mehr oder weniger beliebigen Anwendung des Ausschlussgrundes nach Art. 53 lit.b AsylG argumentativ und praktisch der Weg geebnet.124 Hierbei steht jedoch nicht einzig das «unvermeidliche Spannungsverhältnis von Freiheit und Sicherheit»125 im Fokus, welches vorliegend aufgrund der - im Vergleich zum Strafverfahren - prozessualen Schlechterstellung im verwaltungsrechtlichen Verfahren weiter verstärkt wird. Vielmehr stehen die Argumentationslinien in BVGE 2018 VI/ 5 zusätzlich im Widerspruch zum Schutzgedanken des Asyls an sich. Deshalb und angesichts der zahlreichen gravierenden rechtlichen und tatsächlichen

\footnotetext{
124 Vgl. Frehner/Hruschka (Fn. 29), S. 47.

125 Huster Stefan/Rudolph Karsten: Vom Rechtsstaat zum Präventionsstaat, Frankfurt am Main 2008, S. 12.
} 
Mängel des Urteils sollte sich die darin enthaltene Argumentationsweise nicht zur Praxis festigen dürfen. 